\title{
Análisis de la construcción de identidad profesional en profesores excelentes: encontrando al SER formador de docentes*
}

\author{
Analysis of professional identity construction in excellent \\ teachers: encountering BEING a teacher of educators.
}

Samuel Pérez Norambuena** , Enrique Rivera García***

\section{RESUMEN}

Este estudio es llevado a cabo desde el paradigma interpretativo con una metodología cualitativa, y tiene por objetivo comprender las trayectorias y biografías de siete docentes universitarios, dedicados a la formación del profesorado en una facultad de educación de una universidad chilena, quienes han sido percibidos y considerados como "excelentes" por sus estudiantes. Mediante entrevistas en profundidad y análisis de narrativas, se ha develado el recorrido que han transitado en sus experiencias y vivencias como personas y como docentes.

Los principales resultados nos muestran que estos profesores se han construido en un ir y venir de experiencias, situaciones y circunstancias, evaluadas de distintas maneras, y que han enriquecido su vida personal contribuyendo a fortalecer su labor profesional. También indican que su construcción como docentes ha tenido que ver con sus primeras experiencias como niños y niñas mediante el juego; la presencia de situaciones cotidianas que incidieron en su vocación temprana y en su gusto por la enseñanza, y el contacto con buenos profesores, en distintos momentos de sus vidas, han contribuido significativamente en su identidad profesional y compromiso con la formación de las futuras y futuros docentes.
Palabras clave: formador de docentes, profesor de enseñanza superior, biografía, discurso, narración.

\footnotetext{
* Estudio forma parte del proyecto de investigación interno de la Universidad del Bío-Bío, denominado, "Quienes son y que hacen los buenos profesores que forman futuros profesores"

** Doctor en Educación, Universidad del Bío-Bío, nacionalidad chilena. Contacto: sperez@ubiobio.cl Orcid: https://orcid.org/0000-0002-1710-328X

*** Doctor en Educación Física, Universidad de Granada, nacionalidad española. Contacto: erivera@ugr.es Orcid: http://orcid.org/0000-0002-7748-1741
} 


\section{SUMMARY}

This study rises from the interpretative paradigm with a qualitative methodology. It aims to follow the experiences and biographies of seven university professors who train future educators in a Chilean university's education department. Also, the faculty studied are perceived and considered as "excellent" by their students. Through in-depth interviews and analysis of narratives, we have unveiled the journey they have gone through in their experiences and experiences as individuals and as teachers. The main results show us that these teachers have been built in a coming and going of experiences, situations, and circumstances, evaluated in different ways, enriching their personal lives and contributing to strengthening their professional work. They also indicate that their construction as teachers has had to do with their first experiences as children through play; the presence of daily situations that influenced their early vocation and their taste for teaching, and the contact with good teachers at different times in their lives have contributed significantly to their professional identity and commitment to the training of future teachers.
Key words: teacher trainer, higher education teacher, biography, discourse, narrative. 


\section{Introducción}

La educación está, actualmente, junto con otras demandas sociales, en el centro del debate nacional e internacional. Temas como su financiamiento, el papel que concierne al Estado, los niveles de segregación escolar y la calidad de la educación son parte de los desafíos que presenta este sector. Un tema que ha sido foco de preocupación, permanentemente, ha sido el de la formación inicial de docentes (en adelante FID), ya que, para el logro de los aprendizajes escolares y la calidad de la educación, esta es muy importantes (Bruns \& Luque, 2014).

Dicha preocupación queda demostrada con una serie de reformas educativas en el ámbito universitario, en diversos países, que buscan avanzar en la eficacia de la formación de pedagogos. Por ejemplo, en el contexto europeo, la Declaración de Bolonia, de 1999, y la Declaración de Berlín, de 2003, desencadenaron diversos cambios en dichos procesos.

Sin embargo y pese a su importancia, no existen consensos mínimos orientadores en cómo debe ser la formación de futuros profesores (Rufinelli, 2016). Esto debido a que son varios los factores que influyen en dicha formación. Uno muy importante, poco estudiado y relevado al lugar que corresponde, tiene que ver con el profesor que forma futuros formadores (Vaillant, 2006; Sandoval, 2009; Lozano 2016; Hirmas, 2014). Dentro de esta categoría, quisimos acercarnos a "profesores excelentes", esto por la enorme relevancia e influencia que tienen en la vida de sus alumnos, en el desarrollo de habilidades sociales y en la mejora del autoconcepto (Jordan \& Codana, 2019).

La labor formadora de los profesores en las universidades ha transitado, en el último tiempo, hacia espacios de reconfiguración de lo que se entiende por enseñanza y aprendizaje. En ese contexto, los buenos formadores necesitan ser escuchados y valorados, porque son ellos los que encarnan en su quehacer, desempeño y discurso la formación de profesores; quienes comprenden de mejor manera los desafíos que requiere esta misión socioeducativa, lo cual les permite hablar con propiedad sobre lo que significa ser profesor y los alcances formativos necesarios.

El objeto de estudio de este artículo se centra en ese actor un tanto olvidado en las políticas de FID, el buen profesor de la formación 
inicial docente: quiénes son y cómo se han construido quienes tienen una enorme influencia pedagógica en las futuras estrategias en el aula de los futuros maestros (Ní Chróinín \& O'Sullivan, 2014). Para lograrlo, nos acercamos a sus biografías, sus vidas públicas y privadas, su formación y su desarrollo como especialistas en la enseñanza de pedagogía.

El buen docente universitario es un factor clave en el desarrollo de futuros profesionales (Puente, 2019). Existe evidencia de que una dirección eficaz de las actividades pedagógicas por parte del profesor, su intervención firme y presencia constante, favorecedoras del diálogo y la construcción colectiva de los saberes, permiten lograr aprendizajes universitarios (Prados, et al., 2010).

Las investigaciones sobre este actor han buscado definir principalmente las características que presenta, y que son valoradas por los estudiantes para ser reconocido como "excelente", en las que es posible identificar la presencia de tres grandes competencias: pedagógicas, genéricas y disciplinares (Casero, 2010; Fernández \& González, 2012), expuestas en la siguiente tabla:

Tabla 1

Estudios sobre buenos profesores

\begin{tabular}{lll}
\hline Competencias & Características & Autores \\
\hline $\begin{array}{l}\text { Competencias buenos docentes } \\
\text { pedagógicas }\end{array}$ & $\begin{array}{l}\text { como la comunicación, } \\
\text { claridad al explicar, } \\
\text { organización, fluidez de la } \\
\text { clase }\end{array}$ & $\begin{array}{l}\text { Jerez et al. (2016), Marín et } \\
\text { al. (2011), Merellano et al. } \\
(2016), \text { García y Medecigo, } \\
(2014)\end{array}$ \\
\hline \multirow{2}{*}{$\begin{array}{l}\text { Competencias } \\
\text { genéricas }\end{array}$} & $\begin{array}{l}\text { Relacionadas con } \\
\text { las características } \\
\text { personales, actitudinales e } \\
\text { interpersonales }\end{array}$ & $\begin{array}{l}\text { Pérez y Linzmayer (2018), } \\
\text { (2019), Navia y Hirsch } \\
(2015)\end{array}$ \\
\hline \multirow{2}{C}{$\begin{array}{l}\text { Competencias } \\
\text { disciplinares }\end{array}$} & $\begin{array}{l}\text { Hacen referencia a los } \\
\text { conocimientos en la } \\
\text { materia y la disciplina que }\end{array}$ & $\begin{array}{l}\text { Singh et al. (2013), Casero } \\
\text { (2010) }\end{array}$ \\
\hline
\end{tabular}

Fuente: Elaboración propia.

Se observa que la preocupación por los buenos profesores ha sido principalmente en términos de definir sus características y sus com- 
petencias, ya que las universidades quieren contar con la presencia de profesores de calidad, que asuman los desafíos formativos de manera adecuada.

Este estudio original se acerca a esos buenos docentes desde otra perspectiva: la de sus historias y experiencias. Se acerca a cómo han aprendido y adquirido su conocimiento profesional. Esto beneficiará, sin duda, a una mayor sistematización teórica sobre cómo aprenden los profesores (Russel, Fuentealba e Hirmas, 2016).

Se pretende, a partir de los nuestros hallazgos, la comprensión de ciertos ámbitos que no necesariamente son tenidos en cuenta en las políticas educativas respecto de la formación inicial, tampoco en la formación continua de los docentes activos, ni en los indicadores de calidad y desempeño docente. A propósito de esto, Berry (2007) indica que "la investigación personal de la propia práctica como formador de docentes es un medio importante para una mejor comprensión y mejora de la relación entre la enseñanza y el aprendizaje sobre la enseñanza" (p. 93).

Connelly y Clandinin (1994) afirman que el formador " enseña lo que él es", de modo que el aprender a enseñar no consiste solo en aprendizajes en espacios formales, teorías y modelos de enseñanza, sino en comunicar un cúmulo de experiencias y conocimientos de la vida y de los contextos en los que se ha desenvuelto como persona, estudiante y profesor. Es decir, el aprendizaje está atravesado por el cuer-po, las relaciones y los afectos (De Riba \& Rivelles, 2019).

Nuestro enfoque de investigación se apoya en el biográfico narrativo, que nos permitirá establecer un contacto más directo y profundo entre la teoría y la práctica, especialmente si consideramos que los hechos sociales no existen por sí mismos. Así como el individuo construye un hecho social, es construido a la vez por éste, afectándolo. Es importante reconocer que nuestra "historia" individual se encuentra en un momento particular y situado, sujeto a condiciones pasadas y presentes de posibilidad y restricción (O’Brien \& Rudd, 2019).

Estamos frente una estrategia, para que el profesorado se haga visible y muestre la generación de su saber profesional (Bolívar, 2014). En el relato, el sujeto expone su mirada sobre su propio ser y hacer, permitiendo un acercamiento a su mundo interior, develando ideas y sentimientos. Al hacerlo, el ser humano experimenta el aprendizaje, 
la capacidad de vivir con su historia, alcanzando potencialmente una mayor capacidad de comprenderse a sí mismo como persona y como ser-en-el-mundo (Trezzi et al., 2019).

El relato que hace el sujeto sobre hechos específicos de su vida, puede ser un vehículo para la reflexión crítica, que permita explorar creencias, valores, percepciones y autoidentidad (Spector-Mersel, 2017).

Escuchar historias de buenos profesores otorga la posibilidad de develar los recorridos humanos y profesionales que han colocado al servicio de la formación y al conocimiento de la enseñanza, "constituyen un nuevo marco de pensamiento para el estudio de las prácticas de enseñanza" (Litwin, 2012, p. 26). Estudiar los relatos de buenos docentes puede llevarnos a conocer y comprender buenas prácticas de enseñanza, y, por qué no, a dar luces a los formadores acerca de cómo enfrentar esta labor.

Coincidimos con Connelly y Clandinin (1995) en que "la razón principal para el uso de la narrativa en la investigación educativa es que los seres humanos somos organismos contadores de historias, organismos que, individual y socialmente, vivimos vidas relatadas" (p. 11).

La investigación narrativa sobre cómo se construyen los formadores presenta cuatro principales líneas de estudio: a) investigación en las formas particulares de aprendizaje de los estudiantes; b) investigación focalizada sobre las acciones de los maestros; c) investigación con maestros que examinan el pensar de otros maestros y e) investigación realizada por maestros (Clark, 2001).

Algunos estudios sobre narrativas de docentes universitarios invitan a conocerse y analizar sus formas de liderar los procesos formativos, entre los cuales destacamos el de López (2010) que, entre sus principales hallazgos, indica que estos se construyeron a partir de la "constitución familiar de apoyo", la "admiración por 'alguien' determinante en sus vidas", y los "ambientes letrados". Passarinho (2019) muestra que esas narraciones dan cuenta del "saber personal de los docentes", "saber de la formación escolar", "saber de la formación académica y "vidas que cuentan el conocimiento"; mientras que Proasi y Escujuri (2016) afirman que, en la construcción como docente, se hace presente "el mundo ligado a la vida personal" y "el mundo ligado a la vida profesional". 
Los estudios anteriormente expuestos dan cuenta de los conocimientos adquiridos en trayectorias de vida y cómo éstas han influido en la manera de enfrentar y guiar los procesos formativos. En dichos estudios se destaca lo que Connelly y Clandinin (2006) expresaron como tres lugares comunes de experiencia: temporalidad, sociabilidad y lugar.

Nuestro objeto de estudio se focaliza en la figura del docente y en el relato de su propia experiencia, en aspectos relevantes de la trayecto-ria personal y profesional que les han permitido abrir nuevos sentidos y posibilidades a la existencia como docentes (Contreras, Quiles-Fer-nández \& Paredes, 2019). Promueve a su vez el valor de las narrativas docentes y de las experiencias, las que pueden ser utilizadas y tenidas en cuenta en la FID. Objetivo fundamental de la investigación es comprender el recorrido transitado para llegar a ser considerados "buenos formadores" que intervienen en la FID. Específicamente, el estudio buscó develar la práctica de formación de docentes llevada a cabo por estos actores educativos y, además, entender cómo sus experiencias los han llevado a construirse como formadores de futuros docentes de calidad.

\section{Métodos y técnicas}

Este estudio se enmarca en el paradigma cualitativo abordado bajo el método fenomenológico, que permite el encuentro del ser humano consigo mismo y otorga "la posibilidad de analizar el fenómeno o la ex-periencia significativa que se le muestra a la conciencia" (Fuster, 2018, p. 2). Este enfoque ha posibilitado el acceso al sentido de la experiencia vivida como persona $\mathrm{y}$ profesor, a su historia, a sus interacciones con el mundo y a su práctica pedagógica.

Se utilizó como técnica la entrevista en profundidad, a partir de un guion constituido por las áreas temáticas que se deseaba explorar.

Previamente, para la selección de los docentes, se utilizó un cues-tionario simple en el que se solicitaba al alumnado del último año de pedagogía, de distintas carreras impartidas por esa universidad, que indicaran él o la docente que consideraban como "excelentes forma-dores" y que más habían impactado en su construcción como docen-tes en formación. Una vez recabada la información y seleccionados los docentes (uno por especialidad), se procedió a la realización de las diferentes entrevistas en profundidad, 
que permitieron "encuentros dirigidos hacia la comprensión de las perspectivas que tienen los in-formantes respecto de sus vidas, experiencias o situaciones, tal como las expresan con sus propias vidas (Taylor \& Bogdan, 1996, p. 100).

\section{Criterios de selección de los participantes}

Respecto de los criterios de inclusión de los participantes, estos fue-ron: profesores que forman futuros profesores y buenos profesores se-leccionados por estudiantes. El caso lo componen siete profesores, uno por cada especialidad: Pedagogía en Educación Matemática, Pedagogía en Educación Física, Pedagogía en Inglés, Pedagogía en Historia y Geo-grafía, Pedagogía en Castellano y Comunicación, Pedagogía en Ciencias Naturales, y Pedagogía en Educación Básica, caracterizados en la tabla 2.

Tabla 2

Atributos de los participantes

\begin{tabular}{llllllll}
\hline Participantes & $\begin{array}{l}\text { Docencia } \\
\text { No Univ. }\end{array}$ & $\begin{array}{l}\text { Docencia } \\
\text { Univ. }\end{array}$ & Doctorado & $\begin{array}{l}\text { Estudio } \\
\text { Padres }\end{array}$ & $\begin{array}{l}\text { Estudios } \\
\text { Pregrado }\end{array}$ & $\begin{array}{l}\text { Estudios } \\
\text { Postgrado }\end{array}$ & Sexo \\
\hline Cafarena & 5 años & 15 años & Sí & Básico & Humanidades & Educación & $\mathrm{M}$ \\
\hline Dinator & 10 años & 35 años & No & Básico & Ciencias & Educación & $\mathrm{M}$ \\
\hline Guerín & 11 años & 7 años & No & Medio & Humanidades & Educación & $\mathrm{F}$ \\
\hline Jara & 8 años & 5 años & No & Superior & Básica & Educación & $\mathrm{F}$ \\
\hline Mansilla & 12 años & 8 años & Sí & Superior & Cs. Sociales & Educación & $\mathrm{F}$ \\
\hline Mistral & 10 años & 13 años & Sí & Básico & Humanidades & Educación & $\mathrm{F}$ \\
\hline Vicente & 5 años & 25 años & No & Básico & Cs. Sociales & No & $\mathrm{M}$ \\
\hline
\end{tabular}

Fuente: elaboración propia.

La tabla 2 permite relacionar el nivel sociocultural de estos profesores con los estudios de sus padres, y con ciertas dificultades económicas y de apoyos escolares que presentaron en sus vidas. Otro elemento interesante es que todos hicieron clases antes, en promedio siete años, lo que indica que esa experiencia es relevante a la hora de hablar de buenos profesores en el nivel superior. Además, son profesores que llevan, en promedio, 20 años formando profesores. Esto quiere decir que los años de aula universitaria son relevantes para alcanzar un buen desempeño como formadores de futuros profesores.

Es importante resaltar que todos los estudiados son profesores de profesión, lo que nos indica que, para la formación de profesores, es relevante ser profesor. 
Para el análisis fenomenológico se aplicó la propuesta de López (2010): a) descripción y codificación: función heurística consistente en comprender las narraciones más allá de sus primeras significaciones; b) inferencia: procedimiento intermedio entre la descripción de los datos para transformarlos en información relevante, y c) interpretación de los datos para transformarlos en información relevante. Para dicho análisis se utilizó del software cualitativo NVIVO, a partir de la identificación de las categorías emergentes.

Desde la propuesta anteriormente expuesta, fue posible definir cinco categorías de análisis, guiados por los estudios de Tardif (1999), López (2010), Proasi y Escujuri (2016) y Passarinho (2019), los que fueron analizados cualitativamente. En la imagen 1 podemos apreciar el sistema de categorías construido a partir del discurso de los participantes. En ésta aparecen cuatro ejes que marcan las direcciones que posteriormente vamos a utilizar para realizar la descripción e interpretación de los datos.

\begin{tabular}{|c|c|c|}
\hline & Acceso rénido & Nodos \\
\hline & Archivos & t Nombre \\
\hline & Memos & E) Aprendizajes familiares \\
\hline & Nodos & Juegos en la niñez \\
\hline - 8 目 & Ontet t & Soporte Afectivo-emocional \\
\hline & Archivos & E. Experiencias en la Escuela \\
\hline & का Clasificaciones de archivo & Costes de la profesión \\
\hline & Eienentos externos & L. Desafios escolares \\
\hline 10 & Coddigos & E. Formación de profesores \\
\hline & Nodos & Ambiente de aprendizaje \\
\hline & os Sentimiento & - Compromiso con la formacion docente \\
\hline & 4. Relaciones & L. Vinculacion Teoria y Practica \\
\hline & comes & E. Vocación de profesor \\
\hline 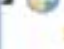 & Casos & Inicio de la vocación \\
\hline & ID Clasificaciones de casos & (1) Profesores comprometidos \\
\hline m & Notas & (1) profesores que marcan \\
\hline$=9$ & Buscar & \\
\hline$\$ 4$ & Mapas & \\
\hline 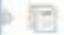 & Salida & \\
\hline
\end{tabular}

Imagen 1: Sistema de categorías utilizado.

Fuente: elaboración propia. 
Descripción de los hallazgos encontrados: develando la mirada de los participantes

A continuación, se dan a conocer las categorías y subcategorías (figura 1) que definen el recorrido realizado por estos docentes, que dan cuenta de la relación de hechos, en apariencia aislados, y que el tiempo se ha encargado de aglutinar como "por arte de magia" en la realidad de hoy:

Desde pequeña, fíjate, le enseñé a las muñecas. Yo creo donde crecí, el entorno en los veranos mis papás planificaban, yo me acuerdo que les ayudaba a planificar, y nos sentábamos los tres con un cuaderno. Tengo que haber hecho puras rayas, después le ayudé a hacer pruebas, a corregir pruebas, siempre me gustó enseñar. (Jara)

La cita anterior nos muestra cómo los hilos narrativos de la primera infancia fueron importantes para lo que hoy son: formadores. De cierta forma, los acontecimientos pasados permiten explicar, en parte, el presente, que, en este caso, tiene que ver con las actuaciones de estos docentes valoradas en una comunidad educativa que forma educadores.

A continuación, se desarrollarán las categorías centrales, para discusión y análisis.

\section{Aprendizajes familiares}

Cuando emerge la nostalgia familiar y se remontan a su niñez y adolescencia, los docentes abren la puerta a sus juegos, vivencias y hogar, al lazo familiar y a lo relevante que fueron para su posterior construcción como personas y como docentes.

Algunos vivieron con sus padres, otros sin alguno de ellos, por separaciones, pérdidas u otros motivos. Independiente de esto, tienen en común algún grado de precariedad económica; sin embargo, no fue algo que ellos recuerden como traumático, sino con un peso emocional generado al darse cuenta hasta dónde han llegado hoy, pese a esas condiciones iniciales:

Durísimo, mi madre era auxiliar de escuela, de las que barrían, me entiendes o no, y yo le ayudaba a barrer (...), mi padre no, porque mi padre no vivía con nosotros, aportaba muy poco y yo comía el almuerzo ahi, en la escuela. Me acuerdo de los garbanzos, los poro- 
tos, las lentejas, porque había unos fondos inmensos y eso comía yo, porque ella tenía derecho a almorzar ahí. (Dinator)

Pese a esas carencias económicas y a las estructuras de familia en que crecieron, existió un soporte afectivo y emocional importante, en ocasiones sus madres, padres, abuelas y tías, permitiéndoles enfrentar los desafíos cotidianos:

...en la niñez mi papá, por trabajo, no siempre estuvo, estuvo muchas veces destinado a distintos lugares, pero estaba mi mamá y mis abuelos paternos, porque además había como un cariño. (Guerín)

Además de esa contención, la presencia del juego como elemento socializador y como una proyección futura inconsciente, que albergó sensaciones y momentos, incidiendo, según ellos, en la elección de ser profesores y guiar personas.

cuando llegaba a la casa, jugaba a hacer profe (...) como tenía cinco hermanos (...) de niño tuve una alta valoración por la escuela, los profesores, me gustaba mucho eso, y entonces jugaba con mis hermanos a ser profe. (Vicente)

\section{Profesores memorables}

En las experiencias escolares vividas como estudiantes, compañeros, en las escuelas, en distintos niveles educacionales y en la universidad, hablan con mucho cariño, admiración y agradecimiento de algunos profesores que pasaron por sus vidas, en los que destacan sus valores, comportamientos y la huella, profunda y duradera, que dejaron en ellos y sus vidas. Inclusos estos profesores afectaron sus elecciones futuras como profesionales de la educación y como personas.

...llegué a estudiar Pedagogía en Historia y Geografía, pero ipor qué?, porque estaba este recuerdo, de la profesora que había sido profesora de historia y geografía, que me enseñó la historia y la geografía, me gustaba la historia y geografía en la escuela básica, la Fresia Segura. (Guerín)

Al recordar su paso como estudiantes, espontáneamente hablan de esos profesores trascendentes. Traen rápidamente a algunos de ellos que les marcaron. Esa huella indeleble quedó almacenada en su memoria afectiva y cognitiva, que aún hoy genera emociones al recordar a estas personas fundamentales, que guiaron sus aprendizajes y creci- 
miento. De alguna manera, la conducta de esos profesores modeló sus actuaciones y maneras de ser actualmente:

...ahi a mi me empezó a gustar la didáctica, porque ahí le encontré asunto, por la manera en la que él enseña; es cómo yo voy a enseñar, y descubríque yo iba a enseñar como la manera que él enseñaba y lo aprendí muy bien. (Mansilla)

\section{Gusto por enseñar}

La enseñanza es un elemento que está siempre presente en sus vidas. Primero a través del juego, luego con experiencias satisfactorias con profesores que iban más allá de lo estipulado en la relación profesor estudiante, lo que marcó sus futuros y la decisión de enseñar a otros. La gran mayoría se hizo profesor antes de ejercer la profesión. Hicieron un recorrido previo lleno de experiencias:

...cuando yo quise ser profesor, así conscientemente, fue en Bulnes, en sexto básico, ahi un profesor un día me dijo: "tú vas a ser profesor". (Vicente)

\section{El desafío de ser profesor en la escuela}

El paso a ser profesor de escuela o liceo fue el momento que habían estado esperando hace mucho tiempo, la gran mayoría desde pequeños. En ese acto se hicieron presentes sus historias como estudiantes, como ayudantes y sus relaciones con esos profesores que fueron importantes. Transformarse en profesor o profesora generó muchos desafíos personales y profesionales, los cuales produjeron alegrías, frustraciones, encantos y desencantos. Estas vivencias fueron muy importantes para consolidar su identidad profesional y permanencia en esta profesión. Esta idea queda patente en la intervención de Cafarena, cuando nos relata que, con 22 años, en el inicio de su desarrollo profesional tiene que asumir una jefatura de "un curso nuevo que se había armado, eran los niños que nadie quería” (Caffarena).

Para muchos de ellos, recordar su paso por la escuela les genera algunos momentos de mucha incertidumbre y ansiedad:

...me generó conflicto, por ejemplo, el rol del profe en ese contexto (...); había mucho ausentismo y uno tenía que ir a las casas, una vez tuve que sacar de un bar a una niña y eso me generó mucho choque, 
y otro chico que estaba con problemas también de drogas (...); uno se decía ¿estaré haciendo lo correcto? (Mansilla)

Estos profesores, comprometidos y responsables con su labor y con sus estudiantes, relatan que asumir esa postura ética con su profesión viene de la mano de algunos costos personales y familiares. Pero estos costos no tuvieron problemas en asumirlos, porque veían que el fin, sus estudiantes, era superior:

...y y me llevaba cuestiones para el fin de semana y de repente no había fin de semana, entonces ahi entraban las discusiones, pero uno va cediendo y vas viendo si te colapsa mucho, necesitas de un compañero que comprenda que es tu vida y que amas lo que estás haciendo. (Mansilla)

\section{Formando profesores para Chile}

La experiencia en formación inicial de profesores, a diferencia de los otros relatos de vida, es en presente, es sobre lo que viven, sienten y promueven actualmente. Hoy, en esta etapa, hacen cuerpo, unidas, aglutinadas, las experiencias de sus vidas. Esta función la asumen con un gran compromiso y responsabilidad, no solo con sus estudiantes y sus procesos, sino que con algo más profundo y simbólico: "yo siempre pienso: estoy formando los futuros profesores para Chile" (Guerín).

Esta etapa ha sido un enorme desafío, ya que nadie estudió para ser profesor universitario; se encontraron con esta posibilidad, por los estudios de posgrado. Algunos lo buscaron y otros sin querer, y han tenido que aprender a formar profesores, con lo que significa eso.

Este espacio de actuación profesional, en educación superior, ha generado un discurso formativo pedagógico y una identidad profesional en la que resaltan la importancia de la formación humana en sus estudiantes.

...estamos formando personas y, la verdad, nuestros estudiantes que llegan dejan o tienen muchas cosas al debe, desde el punto de vista de la formación de valores, del punto de vista de cómo actuar y comportarse en distintas situaciones. Y si no lo hacemos nosotros, ¿quién lo va a hacer? (Guerín)

También les importa la formación profesional de estos futuros profesores: 
...quiero que ellos sean buenos profesores, que manejen sus materias, porque nadie puede entregar lo que no tiene. (Caffarena)

Estos profesores frecuentemente hablan, de manera consciente o no, de la importancia de generar climas adecuados para que se favorezcan los aprendizajes que requieren sus estudiantes. Las características de estos ambientes se relacionan con

entregar lo mejor a los estudiantes, de que ese ambiente sea el más grato, donde el estudiante se sienta a gusto, no que se sienta ;ahhh de nuevo tengo con este profesor! (Mistral)

Además, su compromiso está con eliminar estructuras autoritarias y rígidas en sus desempeños, e incorporar en la formación

metodologías activas, participativas, trabajos en grupos; esto mismo del uso de la tecnología me sirve mucho, porque, por ejemplo, hay un recurso que ellos van respondiendo en tiempo real. (Jara)

Ellos, sin excepción, tuvieron experiencia en la escuela antes de ejercer como docentes universitarios. Ese hecho los lleva a tener presente, en el acto educativo, la relación de sus enseñanzas con la aplicación al aula. Es decir, estos formadores, cuando planifican la enseñanza, lo hacen pensando en sus estudiantes y sus futuros. Ese plan y la didáctica utilizada son coherentes con la búsqueda de profesionales de la educación que promuevan espacios de aprendizaje en la escuela:

...me pregunto cómo se vincula esto con su vida también y (...) después siempre pensar cómo él podría llevar esto al aula. (Dinator)

Esa vinculación la ven como un paso esencial para que sus estudiantes sean profesores de calidad:

...entonces eso espero, que sean buenos profesores, y siempre les digo "si no van a ser buenos profesores en inglés trabajen en otra cosa". (Mistral)

\section{Interpretación de los hallazgos; construyendo teoría sustantiva}

Las experiencias de estos buenos docentes que lideran procesos formativos, expresadas en las narraciones, fueron relatadas a partir de la autocomprensión de los sucesos vividos y de la concepción que tienen de sí mismos (Kelchtermans, 2009). En ese contexto, a continuación, presentamos los siguientes hallazgos. 
Podemos ver que todos ellos, al relatar sus vidas, se han visto enfrentados a diferentes contextos, desafíos, dificultades y quiebres en el tiempo, lo que ha dado forma a lo que son y en lo que se han convertido. Es decir, los contextos personales de los docentes son fundamentales a la hora de comprender el conocimiento del profesor (Richmond, 2016; Avraamidou, 2016; Clandinin, Schaefer \& Downey, 2014).

Estos buenos profesores, al hablar de sus vidas y de su labor formativa, dejan en claro que serlo no es una construcción de un día para otro, sino, más bien, un largo recorrido que, en algunos casos, comienza en la niñez y que abarca mucho tiempo, además de estudios y desgaste físico, emocional y, en ocasiones, familiar. Incluye además experiencias personales con niños y jóvenes, en distintos contextos. Lo que concuerda con lo expresado por Connelly y Clandinin (2006), respecto de que las vidas de los formadores de docentes están íntimamente entrelazadas con las vidas de maestros, niños y jóvenes.

Un resultado muy interesante de este estudio es el valor que estos formadores otorgan a los juegos de la niñez. Han sido rememorados y expresados como vitales para elegir a qué dedicarse en el futuro. Esos juegos y los roles asumidos, como estudiantes o profesores, aparentemente insignificantes, dan cuenta de que se hicieron maestros en tempranas edades, soñando y conectándose con las sensaciones producidas en esos tiempos. Lo que se relaciona con lo dicho por los autores Michnick, Hirsh-Pasek y Singer (2006), en relación con que el juego es una actividad instintiva e imitativa, y prepara las capacidades para la vida adulta, por lo tanto, es inherentemente un proceso de aprendizaje. Achavar (2019) también lo destaca, en términos de que entonces se practican mecanismos de imitación y contrastación con la conducta real, lo que es una fuente de discernimiento de los requerimientos de la vida adulta.

Otorgan estos formadores un valor importante a sus experiencias escolares en etapas iniciales. Se encontraron con maestros que inculcaron afectivamente la responsabilidad y el compromiso con lo que hacen, y el estar al servicio de otros. La mayoría de ellos se dedicó a la enseñanza por esos modelos iniciales, que generaron interés en la especialidad a la que se dedican hoy, lo que concuerda con lo expuesto por Haught, Nardi y Walls (2015), y por Chang-Kredl y Kingsley (2014), 
en términos de que los antiguos profesores influyeron en su identidad actual como maestros.

Al mirar retrospectivamente, esas vivencias iniciales han sido puestas en escena en su labor formativa. Han comprendido que su trabajo es, más allá de la entrega de los contenidos - el cual dominan adecuadamente-, presencia e interacción, lo que ha sido significativo para sus estudiantes y, por supuesto, para ellos. Es decir, ser importantes para sus estudiantes en un sentido educativo más amplio, les ha permitido vivir y otorgar sentido e identidad a su profesión.

Al igual que los resultados de López (2010), estos buenos profesores encarnan un enfoque humanista de la educación, que pregona que toda enseñanza debe ser construida sobre la base de una "ética del cuidado". Relatan que su práctica es con un alto sentido de responsabilidad, con los estudiantes y su formación, y con padres y colegas. Sus actuaciones incorporan la dimensión moral y valórica en el proceso formativo. Respecto de esto, Fenstermacher (1990, p. 132) indica que la enseñanza es "una actividad profundamente moral", y Hamilton (2002) y Cornejo (2016) señalan que la formación docente requiere de formadores comprometidos valóricamente.

En la formación de profesores existe un distanciamiento entre la formación inicial y el ejercicio docente (Brouwer \& Korthagen, 2005), y se debe tener en cuenta el mundo en el que se desarrollará la labor docente (Parra, Navarro, Rubio \& Martín, 2020). Estos profesores han sido capaces de impregnar en sus clases, discursos y prácticas, su preferencia por vincular, regular y conscientemente, los contenidos de las clases con la realidad escolar. Esto puede suceder porque, sin excepción, todos tuvieron experiencias como profesores de escuela, y eso les ha dado una identidad profesional que promueve esta conexión.

\section{Conclusiones}

La formación humana, las emociones y los sentimientos, emergen como elementos claves en la construcción de un buen docente. Pero no podemos olvidar que los espacios, los tiempos y las vivencias en los que éste se desarrolla como persona, van orientando y guiando el profesor en el que se convertirá. Será en el receptáculo de estos espacios, tiempos y vivencias, en los que aprenderá la importancia de 
la empatía, el compromiso y la responsabilidad, el amor a la vida y la afectividad como pilares del compartir humano. Valores y principios intransables que, acompañados de un riguroso sentido del "aprender bien para enseñar bien", forman parte de sus principales motivaciones para enseñar.

Se perciben intactas en sus narraciones la motivación y la vocación, pese a los años de trabajo desarrollado en distintos niveles. Además, son inmunes a las influencias externas que condicionan su labor, como leyes y normativas que entorpecen en vez de facilitar, o las actitudes negativas de algunos estudiantes. Lo que permite concluir que estos buenos profesores tienen una enorme fortaleza interna para responder a esas situaciones en el tiempo, y sostener la difícil tarea de formar los futuros profesores para Chile.

Finalmente, nos atrevemos a sugerir, a partir del estudio realizado, cinco claves de actuación a tener presente en la Formación Inicial Docente:

En primer lugar, facilitar a nuestro alumnado experiencias significativas en el ámbito personal, social y profesional; propuestas que le hagan comprender la complejidad que posteriormente encontrarán en los centros educativos.

En segundo término, apostar por un aprendizaje profesional, planteado desde una visión integral y global, que ponga en el centro del proceso al alumnado que aprende y le permita generar las competencias básicas con las que pueda dar respuesta a la complejidad de la escuela.

En tercer lugar, tomar como referente la ética docente. Formar incorporando el desarrollo de valores que impliquen el compromiso personal y de responsabilidad social con las comunidades escolares.

En cuarto, apostar por la necesaria vocación del docente. El alumnado debe identificar que la docencia es compromiso y, por lo tanto, es preciso tener vocación para su desempeño. Para lograr este objetivo debemos, como diría Fernando Savater, educar desde el cuerpo a cuerpo.

Por último, los cambios que se proponen al estudiantado deben venir desde la acción, pero, aún más, desde la reflexión pedagógica. Solo 
desde este punto de partida es posible llegar al aprendizaje dialógico y construir el andamiaje que soporte el futuro desarrollo profesional de nuestro alumnado.

\section{Referencias}

Achavar, C. (2019). Beneficios del juego en la acción pedagógica. Revista Foro Educacional, (33), 115-122.

Avraamidou, L. (2016). Intersections of life histories and science identities: The stories of three preservice elementary teachers. International Journal of Science Education, (38), 861-884. DOI: https://doi.org/10.1080/ 09500693.2016.1169564

Bolívar, A. (2014). Las historias de vida del profesorado. Voces y contextos. Revista Mexicana de Investigación Educativa, 19(62), 711-734.

Berry, A. (2007). A journal of self-study of teacher education practices. "Reconceptualizing Teacher Educator Knowledge as Tensions: Exploring the tension between valuing and reconstructing experience". Studying Teacher Education, 3(2), 117134.

Brouwer, N. \& Korthagen, F. (2005). Can teacher education make a difference? American Educational Research Journal, 42(1), 153-224.

Bruns, B. \& Luque, J. (2014). Excellent Teachers: How to Improve Learning in Latin America and the Caribbean. Washington, DC: Word Bank.

Casero, A. (2010). ¿Cómo es el buen profesor universitario según el alumnado? Revista Española de Pedagogía, 68(246), 223242.

Chang-Kredl, S. \& Kingsley, S. (2014). Identity expectations in early childhood teacher education: Preservice teachers' memories of prior experiences and reasons for entry into the profession. Teaching and Teacher Education, (43), 27-36.

Chávez, G. (2019). Conocimiento, didáctica y empatía: rasgos de los profesores de excelencia según los estudiantes universitarios. En A. Hirsch y J. Pérez (Coords.), Ética profesional y responsabilidad social universitaria: experiencias institucionales (pp. 149-164). Ciudad de México.

Clark, R. (2001). Learning from media. Arguments, Analysis and Evidence. Greenwich, Connecticut: Information Age Publishing. 
Clandinin, D. J., Schaefer, L. \& Downey, A. (Eds.). (2014). Narrative Conceptions of Knowledge: Towards Understanding Teacher Attrition. Bingley, UK: Emerald Group.

Connelly, F. M. \& Clandinin, D. J. (1994). Telling teaching stories. Teacher education quarterly, 21(1), 145-158.

Connelly, F. M. y Clandinin, D. J. (1995). Relatos de experiencia e investigación educativa. En J. Larrosa et al. (comps.), Déjame que te cuente. Ensayos sobre narrativa y educación (pp. 11-59). Barcelona: Laertes/Psicopedagogía.

Connelly, F. M. \& Clandinin, D. J. (2006). Narrative Inquiry: A Methodology for Studying Lived Experience. Research Studies in Music Education, (27), 44-54. DOI: http://dx.doi.org/10.117 7/1321103X060270010301.

Contreras, J., Quiles-Fernández, E. y Paredes, A. (2019). Una pedagogía narrativa para la formación del profesorado. Márgenes, 5875. DOI: http://dx.doi.org/10.24310/mgnmar.v0i0.6624.

Cornejo, J. (2016). El Self-Study de la práctica de los formadores de futuros profesores: bases teóricas, características y modalidades metodológicas. En T. Russell, R. Fuentealba, y C. Hirmas (Comp.), Formadores de formadores, descubriendo la propia voz a través del self-study. Santiago, Chile: OEI.

De Riba, S. y Rivelles, B. (2019). Hacia una pedagogía afectiva del movimiento. Tercio Creciente, (16(, 7-30. DOI: https://dx.doi. org/10.17561/rtc.n16.1.

Fernández, M. y González, S. (2012). El perfil del buen docente universitario: una aproximación en función del sexo del alumnado. Revista de Docencia Universitaria, Valencia, 10(2), 237-249.

Fenstermacher, G. (1990). Some moral considerations on teaching as a profession. In: J. Goodlad, R. Soder \& K. Sirotnik (Eds.), The moral dimensions of teaching (pp. 130-151). San Francisco: Jossey-Bass.

Fuster, D. (2019). Investigación cualitativa: Método fenomenológico hermenéutico. Propósitos y Representaciones, 7(1), 201-229.

García, J. y Medecigo, A. (2014). Los criterios que emplean los estudiantes universitarios para evaluar la in-eficacia docente de sus profesores. Perfiles educativos, 36(143), 124-139.

Haught, P., Nardi, A. \& Walls, R. (2015). Preservice teachers' academic memories of school: A tool for learning. American Journal of Educational Research, 3(2), 166-172. 
Hamilton, M. L. (2002). Change, social justice, and re-liability: Reflections of a secret (change) agent. In: J. Lougrhan and T. Russell (Eds.), Improving Teacher Education Practiques Through Self-Estudy (pp. 176-189). , Falmer, London: Rougtledge.

Hirmas, C. (2014). Tensiones y desafíos para pensar el cambio en la formación práctica de futuros profesores. Estudios Pedagógicos, 40(1), 127-143.

Jerez, Ó., Orsini, C. y Hasbún, B. (2016). Atributos de una docencia de calidad en la educación superior: una revisión sistemática. Estudios pedagógicos, 42(3), 483-506.

Jordan, J. y Codana, A. (2019). La influencia del profesor apasionado en la mejora académica y el desarrollo personal de sus alumnos. Revista Estudios Sobre Educación, (36), 31-51. DOI: https:// doi.org/10.15581/004.36.31-51

Kelchtermans, G. (2009). Who I am in how I teach is the message: self囚 understanding, vulnerability and reflection. Teachers and Teaching, 15(2), 257-272, DOI: 10.1080/13540600902875332

López, S. (2010). Los buenos profesores. Educadores comprometidos con un proyecto educativo. La Serena, Chile: Editorial Universidad La Serena.

Litwin, E. (2012). El oficio de enseñar. Condiciones y contextos. Buenos Aires: Paidós.

Lozano, I. (2016). Las trayectorias formativas de los formadores de docentes en México. Revista Electrónica Actualidades Investigativas en Educación, 16(1), 1-25.

Marín, M., Martínez, R., Troyanor, Y. y Teruel, P. (2011). Student Perspectives on the University Professor Role. Social Behavior and Personality, An International Journal, (39), 491-496.

Merellano, E., Almonacid, A., Moreno, A. y Castro, C. (2016), Buenos docentes universitarios: $i$ Qué dicen los estudiantes? Educação e Pesquisa, 42(4), 937-952.

Michnick, R., Hirsh-Pasek, K. \& Singer, D. (2006). Play= Learning. How play motivates and enhances children's cognitive and socialemotional growth. New York: Oxford University Press.

Navia, C. y Hirsch, A. (2015). Dimensiones y rasgos sobre la excelencia del profesorado en instituciones formadoras de docentes en dos países de América Latina. Edetania. Estudios y propuestas socioeducativas, (48), 117-130. 
Ní Chróinín, D. \& O`Sullivan, M. (2014). From Initial Teacher Education through Induction and Beyond: A Longitudinal Study of Primary Teacher Beliefs. Irish Educational Studies, 33(4), 451-466.

O'Brien, S. \& Rudd, T. In Y. Novakovic and P. Sikes (Eds, 2019). Storying the public intellectual: Commentaries on the impact and influence of the work of Ivor Goodson. London and New York: Routledge.

Parra, G., Navarro, A., Rubio, F. y Martín, B. (2020). Teaching-life Histories: An Analysis of Initial and Continuing Training for Twenty-first century Teachers. Estudios sobre Educación, (39), 159-178.

Passarinho, L. (2019). Leituras de si: saberes docentes e história de vida de formadores de professores, Educar em Revista, (74), 291308.

Pérez, S. y Linzmayer, L. (2018). Los buenos profesores en la mirada de padres y apoderados. Educación y Educadores, 21(3): 373387.

Prados, M., Cubero, R. y De La Mata, M. (2010). What structures do university teachers and students use in their classroom interaction. Electronic Journal of Research in Educational Psychology, 7(1), 163-194.

Proasi, L. y Escujuri, J. (2016). Vida personal y desarrollo profesional de los profesores memorables. El modelo de instalación: una mirada desde el entramado narrativo. Revista EntramadosEducación y Sociedad, 3(3), 135-141.

Puente, K. \& Morillo, Z. (2019). Analysis of university level teacher training: reflections and revisions. Revista Espirales, 3(28), 46-62.

Richmond, G. (2016). Making sense of the interplay of identity, agency, and context in the development of beginning science teachers in high-poverty schools. In L. Avraamidou (Ed.), Studying science teacher identity: Theoretical, methodological and empirical considerations (pp. 219-236). Rotterdam: Sense Publishers.

Rufinelli, A. (2016). Ley de desarrollo profesional docente en Chile: de la precarización sistemática a los logros, avances y desafíos pendientes para la profesionalización. Estudios Pedagógicos, $40(4), 261-279$ 
Russell, T., Fuentealba, R. e Hirmas, C. (Comp.). (2016). Formadores de formadores, descubriendo la propia voz a través del selfstudy. Organización de Estados Iberoamericanos para la Educación, la Ciencia y la Cultura (OEI).Sandoval, E., Harold, I. y Blum, R. (Eds.). (2009). Desafíos y posibilidades en la formación de maestros una perspectiva desde América del norte. México: UPN-The University of New Mexico.

Singh, S., Pai, D., Sinha, N., Kaur, A., Soe, H. \& Barua, A. (2013). Qualities of an effective teacher: what do medical teachers think? BMC Medical Education, 13(128), 1-7.Spector-Mersel, G. (2017). Reflection of life history in social work education: a practical model. Journal of Education in Social Work, 53(2), 286-299. DOI: 10.1080/10437797.2016.1243498.

Tardif, J. (1999). Le transfert des apprentissages. Montréal: Éditions Logiques.

Taylor, S. y R. Bogdan (1996). Introducción a los métodos cualitativos de investigación. Barcelona: Paidós Básica.

Trezzi, C., Romero, M. y May, M. (2019). Narrativas fotográficas: uma contribuição para a formação do profesor. Conhecimento \& Diversidade, 11(24), 91-103.

Vaillant, D. (2006). Atraer y retener buenos profesionales en la profesión docente: políticas en Latinoamérica. Revista de Educación, (340), 117-140. 